\title{
Caso clínico: Anestesia para cesárea en paciente con atresia tricuspidea
}

\author{
Case report: Anethesia for cessarean seccion in tricuspid atresia \\ Yosselly Ninoska Carrillo Aybar1,*, Renato Moreno Gonzales² \\ 1 Residente de Anestesiología del Instituto Nacional Materno Perinatal de Lima, Perú. \\ 2 Anestesiólogo del Instituto Nacional Materno Perinatal de Lima, Perú.
}

Fecha de recepción: 24 de septiembre de 2020 / Fecha de aceptación: 12 de diciembre de 2020

\begin{abstract}
Introduction: In developed countries congenital heart disease is one of the most frequent cardiac diseases in pregnancy, while valvular disease has a higher prevalence in developing countries. In order to perform multidisciplinary management and avoid fatal outcomes it is important to know and review the relevant literature when we take care of a pregnant patient with congenital heart disease. Case description: We describe a case of a 35 year old multiparous pregnant woman, with a diagnosis of tricuspid atresia with perimembranous interventricular septal defect, presented at 31 weeks gestation for an emergency cesarean section for the third time. A low dose sequential combined spinal epidural block technique was performed. First-line uterotonics were used associated with a restricted water balance. During pregnancy, the patient presented central and peripheral cyanosis, nail clubbing, and oxygen saturation of $78 \%$ on room air. In addition, a review of the literature was made. Discussion: It is important to perform risk stratification in pregnant women with congenital or acquired heart disease, and a multidisciplinary management should be carried out preserving the mother-child binomial wellbeing. Neuroaxial anesthesia can be indicated in some cases, with the appropriate use of uterotonics and restricted water balance can improve the outcomes in the management of pregnant women with heart disease. Conclusion: Neuraxial techniques must be individualized. Knowing the pathophysiology of congenital heart disease and cardiovascular physiological changes during pregnancy is important for proper management of this type of patient. A low dose sequential combined spinal epidural block technique was effective and safe in this patient.
\end{abstract}

Key words: Congenital heart disease, pregnancy, combined spinal epidural anesthesia; tricuspid atresia, peri membranous ventricular septal defect.

\section{RESUMEN}

Introducción: La enfermedad cardiaca congénita es una de las más frecuentes en países desarrollados, pero en países en vías de desarrollo tiene mayor prevalencia las enfermedades valvulares, sin embargo, al presentarse una paciente gestante con cardiopatía congénita es importante conocer y revisar la literatura pertinente para realizar un manejo multidisciplinario y evitar tener desenlaces fatales que pueden evitarse. Presentación del caso: Presentamos el caso de una multigesta de 35 años con embarazo de 31 semanas, con el diagnóstico por ecocardiograma de atresia tricuspídea con comunicación interventricular perimembranosa desde su primera gestación, la cual fue intervenida por tercera vez de cesárea de emergencia bajo la técnica combinada espinal epidural variante secuencial. Se usó uterotónicos de primera línea asociado a un balance hídrico estricto. La paciente durante la gestación presentó cianosis peribucal y extremidades, con uñas en vidrio de reloj, saturación de oxígeno de $78 \%$ con fracción inspiratoria de oxígeno ambiental. Además, se realizó revisión de la literatura al respecto. Discusión: Es importante realizar la estratificación de riesgo en gestantes con cardiopatía congénita o adquirida; ya que, según lo establecido, se podrá realizar un manejo multidisciplinario preservando el binomio madre hijo, la anestesia neuroaxial según sea el caso está indicado realizarla, aunado a ello el uso adecuado de uterotónicos y control del balance hídrico permitirá tener un mejor éxito en el manejo de las gestantes con cardiopatías. Conclusión: Las técnicas neuroaxiales deben ser individualizadas. Conocer la fisiopatología de las cardiopatías congénitas y los cambios fisiológicos cardiovasculares durante la gestación es importante para realizar un manejo adecuado en este tipo de pacientes. La anestesia regional técnica combinada variante secuencial fue efectiva y segura en esta paciente.

Palabras clave: Embarazo, anestesia combinada espinal epidural, atresia tricuspídea.

yosselly@hotmail.com

*ORCID: https://orcid.org/0000-0001-7166-8305

ORCID: https://orcid.org/0000-0002-5465-4929 


\section{Introducción}

a enfermedad cardiaca materna durante el embarazo se presenta entre el $1 \%$ a $4 \%$ en los países desarrollados, según el Registro Mundial Prospectivo de Mujeres con Embarazo y Enfermedad Cardiaca (ROPCA: Registry Of Pregnancy And Cardiac disease). La enfermedad cardiaca congénita es la más común en $70 \%$, sin embargo, en los países en vías de desarrollo la enfermedad valvular alcanza $55 \%$ de prevalencia. El $10 \%$ de embarazadas con cardiopatías congénitas presentan complicaciones cardiacas como insuficiencia cardiaca, arritmias, isquemia coronaria y eventos tromboembólicos[1],[2].

Durante el embarazo existen cambios fisiológicos en diversos aparatos y sistemas, a nivel cardiovascular destaca el aumento del volumen plasmático el cual alcanza valores de $40 \%$ $45 \%$ sobre los niveles pregestacionales, determinando de esta manera el aumento del gasto cardiaco del 30\%-50\% con un pico entre las semanas 20 y 24 de gestación; así como también la frecuencia cardiaca aumenta aproximadamente $20 \%$ sobre los niveles basales y la resistencia vascular sistémica disminuyen. Es por ello que las probabilidades de eventos adversos: cardiovasculares, obstétricos y fetales durante el embarazo son mayores en gestantes con cardiopatías congénitas, entre ellas la prematuridad, pequeños para la edad gestacional u otras anomalías congénitas, eventos obstétricos como desprendimiento prematuro de la placenta, placenta previa, preeclampsia, hipertensión en el embarazo y parto prematuro[3],[4].

Existen diferentes clasificaciones de las enfermedades cardiacas congénitas, sin embargo, la clasificación fisiológica es una de las más útiles, ya que determina si la mezcla de sangre predominante es de izquierda a derecha o de derecha a izquierda o una interacción entre las resistencias pulmonares o sistémicas, incluso estas pueden presentarse como lesiones aisladas, más de una lesión o combinadas entre ellas; es el caso de la atresia tricuspídea que es considerada una cardiopatía congénita cianótica con flujo pulmonar disminuido, siendo de manera contraria en la comunicación interventricular que es una cardiopatía congénita a cianótica con flujo pulmonar aumentado[5].

\section{Descripción del caso}

Presentamos el caso de una multigesta de 35 años con embarazo de 31 semanas y diagnóstico de atresia tricuspídea con comunicación interventricular perimembranosa, además del área de la aurícula derecha de $19,8 \mathrm{~cm}^{2}$ y el tiempo de aceleración de la válvula pulmonar de 60 m, por ende, tiene probabilidades intermedias de hipertensión pulmonar por ecocardiograma (Figura 1). Fue diagnosticada de patología cardiaca desde su primer embarazo durante sus controles prenatales. Niega hospitalizaciones previas por patología cardiaca. Niega antecedentes familiares de importancia. Programada para cesárea categoría Dos de Lucas et al., por presentar RCIU y ecografía doppler fetal alterada. Entre los antecedentes obstétricos: dos gestaciones pretérmino de 34 y 31 semanas concluida por cesárea hace 5 y 3 años respectivamente, aparentemente sin complicaciones.

Paciente en regular estado general, zonas de punción venosa accesible, presencia de dedos en palillo de tambor y uñas de vidrio esmerilado de miembros superiores e inferiores con presencia de cianosis central y periférica, ausencia de varices, y presencia de edemas ++/+++ en región lumbosacra, que dificulta la palpación de las apófisis espinosas.

Al examen físico: score de Mallampati III, distancia tiromentoniana de $6 \mathrm{~cm}$, apertura bucal de $3 \mathrm{~cm}$ y subluxación mandibular mayor a 0 grados, adecuado movimiento cervical, tráquea central y dentadura sin alteraciones. A nivel cardiovascular presencia de soplo sistólico en foco tricuspídeo III/IV, ausencia de ingurgitación yugular. Las funciones vitales se describen a continuación: saturación de oxígeno 78\% con una fracción inspiratoria de oxígeno del $21 \%$, frecuencia respiratoria de 18 , frecuencia cardiaca de 85 latidos por minuto y presión arterial de 120/69 mmHg medido en brazo derecho, Escala de Estado Físico ASA III, WHO III y Clase Funcional 2 (Tabla 1); exámenes auxiliares de laboratorio prequirúrgicos en rangos de normalidad, excepto la plaquetopenia leve (Tabla 2).

Paciente usó enoxaparina 40 mg vía subcutánea una sola vez, tres días antes de ingresar a sala de operaciones, luego lo suspendió. Se le explica a la paciente las opciones anestésicas, se decide realizar la técnica combinada espinal epidural variante secuencial. Se realizó monitorización ASA básica, paciente sentada previa antisepsia, se realiza la técnica combinada en diferentes espacios, realizándose habón cutáneo con lidocaína al $2 \%$ sin epinefrina y seguidamente se introduce la aguja Tuohy $N^{\circ} 18$ en el espacio intervertebral L2-L3 determinando el espacio epidural mediante el método de pérdida de resis-

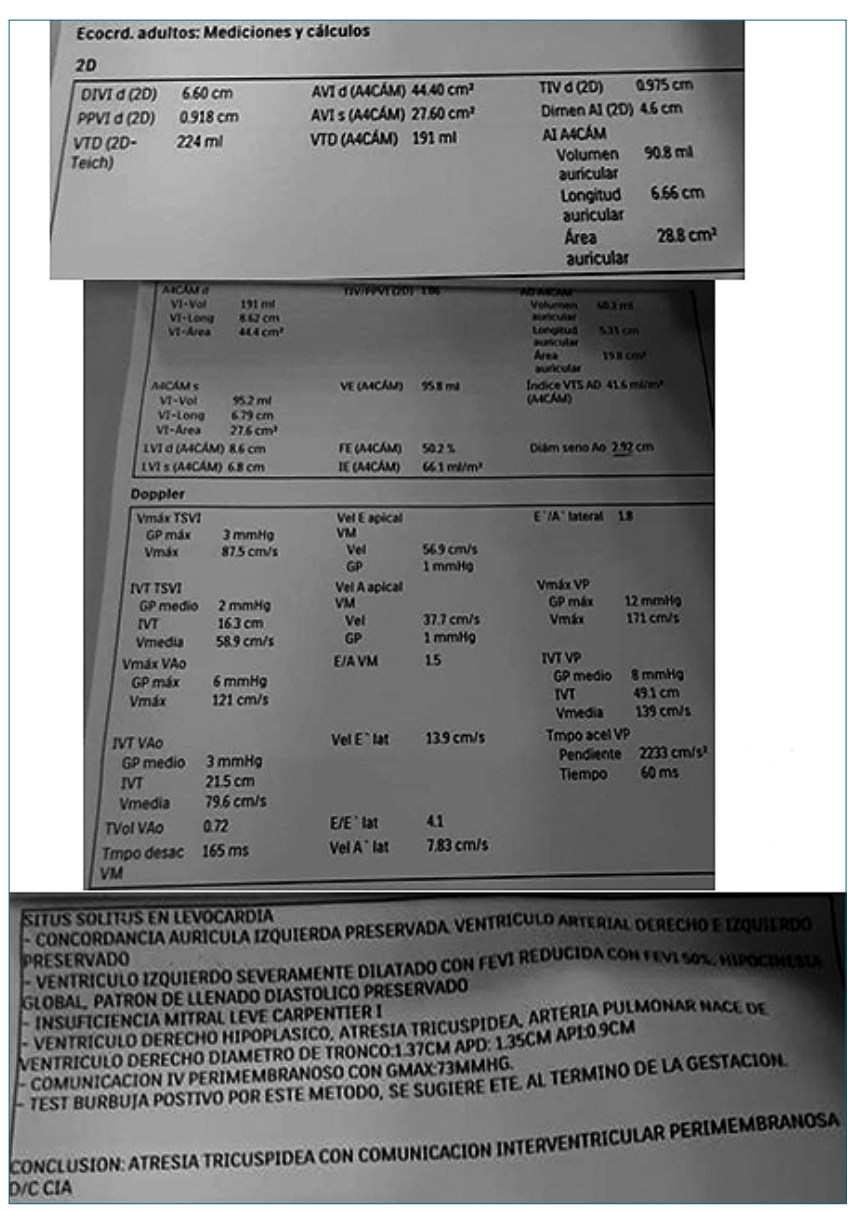

Figura 1. Ecocardiograma. 
tencia intermitente, se deja catéter epidural fijado en $10 \mathrm{~cm}$, sin complicaciones, luego se procede a realizar con la aguja espinal punta de lápiz $N^{\circ} 27 \mathrm{G}$ en el espacio intervertebral L4L5 y bupivacaína hiperbárica 0,5\% 6 mg más fentanilo 20 ug con morfina $100 \mathrm{ug}$, paciente tolera procedimiento. Se coloca en decúbito supino con inclinación lateral en ángulo de $15^{\circ}$, posteriormente, se comprueba nivel anestésico por el método de temperatura que abarcó hasta el dermatoma T8 y a los 7 minutos se adiciona por el catéter epidural bupivacaína 0,25\% 25 mg (5 cc) llegando hasta el dermatoma T3. Paciente con frecuencia cardiaca y PAM estables durante el acto quirúrgico, saturación de oxígeno 78\%-82\% ventilando espontáneamente con fracción inspiratoria ambiental, no se usó vasopresor profiláctico en infusión ni hubo la necesidad de usar bolos manuales de rescate, porque la paciente no presentó hipotensión durante y después de la anestesia. El nacimiento ocurrió a los 6 minutos de inicio de la cirugía con un recién nacido sano apgar 8-9. Se utilizó la primera línea de uterotónicos, oxitocina 3 UI en infusión lenta, luego de tres minutos se repitió $3 \mathrm{Ul}$ en infusión lenta, porque aún no se lograba el tono adecuado del útero, se agregó 3 UI en un cloruro de sodio $9 \%$ de $100 \mathrm{ml}$ para que continúe la infusión durante el acto quirúrgico.

Se infundió $500 \mathrm{ml}$ de cloruro de sodio $9 \%$ durante el intraoperatorio. Para el manejo de la analgesia posoperatoria se administró paracetamol $1 \mathrm{~g}$ por vía endovenosa. Se cuantifico el sangrado intraoperatorio de $500 \mathrm{ml}$ aproximadamente. La paciente paso a la unidad de cuidados Intermedios materno para monitorización con funciones vitales estables, durante la evolución posanestésica, según escala de Aldrete tuvo 10 puntos y según escala de Bromage un puntaje de 0 , transfundieron 2 paquetes globulares, 4 crioprecipitados por presentar $700 \mathrm{ml}$ de sangrado vaginal por hipotonía uterina, con un control de hemoglobina posterior a la transfusión de $13 \mathrm{mg} / \mathrm{dl}$, luego fue desplazada a hospitalización, durante su estancia solo administraron enoxaparina 40 mg por vía subcutánea 24 h después del acto quirúrgico en una sola oportunidad. No presentó ningún evento tromboembólico durante la hospitalización y deciden su alta sin aparentes complicaciones.

\section{Discusión}

En las gestantes con enfermedad cardiaca congénita el manejo debe ser multidisciplinario para disminuir la morbimortalidad materno fetal, siendo necesario prepararse anticipadamente para los riesgos y probables complicaciones que conllevan este tipo de enfermedades cardiacas congénitas ya que la incidencia de mujeres embarazadas con cardiopatía congénita y cardiopatía adquirida está en aumento aproximadamente en $5 \%$ por año[4],[6].

El Consenso Europeo de manejo de enfermedades cardiovasculares recomienda estratificar el riesgo cardiológico antes y durante la gestación (Tabla 1), en esta paciente se estratificó un riesgo WHO III, considerado riesgo moderado en compara-

Tabla 1. Clasificación modificada de la Organización Mundial de la Salud (OMS) para evaluar el riesgo de la gestante con cardiopatía

\begin{tabular}{ll}
\hline Categoría OMSm & Enfermedad cardiaca \\
I & Estenosis pulmonar leve a moderada \\
& Ductus arterioso persistente \\
& Prolapso de válvula mitral \\
& Lesiones simples corregidas exitosamente (CIA, CIV, conexión pulmonar venosa anómala) \\
& CIA o CIV no corregida \\
& Tetralogía de Fallot no corregida \\
& Disfunción leve a moderada del ventrículo izquierdo (fracción de eyección mayor al 30\%) \\
& Enfermedad valvular nativa no incluida en la categoría I o IV \\
& Síndrome de Marfán sin dilatación aortica \\
& Válvula aórtica bivalva con diámetro de la raíz aórtica menos de $45 \mathrm{~mm}$ \\
& Coartación de aorta corregida \\
& Válvula mecánica \\
& Ventrículo derecho sistémico \\
& Circulación de Fontan \\
& Enfermedad cardiaca congénita cianosante no corregida \\
& Enfermedad cardiaca congénita compleja \\
III $\quad$ Síndrome de Marfán con dilatación de raíz aórtica entre 40 y $45 \mathrm{~mm}$ \\
Válvula aórtica bivalva con diámetro de la raíz aórtica entre $45 \mathrm{ym} 50 \mathrm{~mm}$ \\
Hipertensión pulmonar de cualquier causa \\
Disfunción grave del ventrículo izquierdo (fracción de eyección menor al $30 \%$ o clase funcional NYHA III o IV) \\
Estenosis mitral grave \\
Estenosis aórtica grave sintomática \\
Síndrome de Marfán con dilatación de raíz aórtica mayor a $45 \mathrm{~mm}$ \\
Válvula aortica bivalva con diámetro de la raíz aortica mayor a $50 \mathrm{~mm}$ \\
Coartación de aorta grave no corregida
\end{tabular}

CIA: Comunicación interauricular, CIV: Comunicación interventricular; Fuente: Adaptado del Manual Práctico Manejo Anestésico de la Paciente Embarazada; Universidad Pontificia Bolivariana, Medellín. Colombia 2018. 


\begin{tabular}{lcc} 
& Tabla 2. Exámenes de laboratorio prequirúrgicos & \\
\hline & Exámenes prequirúrgicos & \\
Perfil de coagulación & VN: $8-22^{\prime \prime}$ & VN: $22-42^{\prime \prime}$ \\
TP & $12,8^{\prime \prime}$ & VN: $300-600$ \\
Fibrinógeno & $30^{\prime \prime}$ & 272 \\
& Hemograma/bioquímico & 14,8 \\
Hemoglobina & 137.000 & 30 \\
Plaquetas & 0,80 \\
Urea & 92 \\
Creatinina & No reactivo \\
Glucosa & No reactivo \\
Serológico (RPRNDRL) & No tiene \\
COVID-19 (prueba rápida) & \\
RQ cardiológico &
\end{tabular}

Fuente: Historia clínica.

ción con otras patologías cardiacas asociadas al embarazo. La evaluación del riesgo debe incluir historia clínica, examen físico, electrocardiograma y ecografías transtorácica según la complejidad de la patología se solicitarán otros exámenes auxiliares; aquellas que tengan enfermedad congénita se les informará sobre el riesgo de transmisión al hijo y/o complicaciones. Si las gestantes tuvieras lesiones congénitas de alto riesgo y comorbilidades se debe tener en cuenta el desarrollo de complicaciones materno fetales, dentro de los eventos cardiacos más frecuentes, edema pulmonar y arritmias[2],[7],[9].

En relación con las cardiopatías congénitas durante el embarazo, sobre todo la comunicación interventricular, se recomienda valorar las dimensiones del corazón y las presiones pulmonares antes del embarazo. También la saturación arterial de oxígeno $<90 \%$ se considera como predictor cardiovascular materno en presencia de cianosis, que se correlaciona con la presencia de doppler útero placentario anormal, condicionando a restricción de crecimiento fetal, prematuridad y muerte fetal[3],[9].

En cuanto al uso de uterotónicos en cardiópatas, existen múltiples aportes que varían de acuerdo a la experiencia que tuvieron en el manejo profiláctico de hemorragia posparto y los efectos hemodinámicos de la oxitocina, ya que dependerá de la dosis y la tasa de administración, así como la presencia de comorbilidades; aún no existe un consenso determinado, algunos autores mencionan el uso de infusión lenta endovenosa de oxitocina 2 UI durante 10 minutos después del nacimiento, seguida de $12 \mathrm{mU} / \mathrm{min}$ durante $4 \mathrm{~h}$, pero se debe tener un control estricto del balance hídrico ya que se puede hacer una carga innecesaria de volumen después del parto y agravar cuadros de insuficiencia cardiaca congestiva si la paciente lo presentara. En algunas pacientes está indicada de manera profiláctica sutura B-Lynch entre otras técnicas quirúrgicas para prevenir la atonía uterina. También se puede administrar bolos endovenosos de 0,1 a 0,5 UI ya que mostró disminución considerable de la presión arterial, pero transitoria sin efectos adversos. Como segunda línea de tratamiento está recomendado el uso de misoprostol 200 - 1.000 mg, tiene un impacto mínimo en los parámetros cardiovasculares, debe evitarse el uso de análogos de ergometrina y prostaglandina $\mathrm{F}[10],[11]$.

Durante el posparto se debe tener en cuenta que está asociado a cambios hemodinámicos importantes, sobre todo en las primeras 24 a $48 \mathrm{~h}$ que podrían agravar o iniciar un cuadro de insuficiencia cardiaca, es por ello que la monitorización hemodinámica, balance hídrico y diuresis debe ser estricto en este tipo de pacientes, se debe continuar hasta el tercer día del puerperio[9].

Al margen de la técnica anestésica que se decida elegir se debe dar mayor énfasis a un manejo multidisciplinario y realizar una monitorización adecuada durante el intraoperatorio y posoperatorio. Es indispensable que el anestesiólogo conozca el tipo, severidad y progresión de la patología dentro del contexto de los cambios fisiológicos cardiovasculares durante el embarazo, fisiología de la labor de parto, parto y el puerperio. Tener en cuenta que el periodo posparto inmediato hasta el cuarto día del puerperio, el gasto cardiaco aumenta aproximadamente en $40 \%$ poniendo en alto riesgo a las gestantes con cardiopatías congénitas. Se podría administrar analgesia de parto y dosis anestésica si es necesario, sin embargo, debe evitarse la hipotensión, especialmente en lesiones valvulares o función ventricular disminuida, en cuanto al manejo de líquidos endovenoso debe usarse con cuidado[3],[9].

Se decidió realizar la técnica combinada espinal epidural variante secuencial con dosis mínimas en esta paciente, debido a la seguridad y calidad que brinda ésta, ya que causa menos vasodilatación que la anestesia general durante la inducción y el uso de agentes inhalatorios, la anestesia regional brinda analgesia posoperatoria, la paciente ventila espontáneamente, por ello tener en claro las metas hemodinámicas y las metas anestésicas en este tipo de pacientes; durante el posoperatorio se debió considerar la tromboprofilaxis[9],[10]. 


\section{Conclusión}

Durante el embarazo se producen cambios a nivel cardiovascular, si a ello se suma una patología cardiaca materna congénita o adquirida, se debe individualizar y realizar un manejo multidisciplinario, teniendo en cuenta desde la estratificación de riesgo de la gestante hasta el puerperio inmediato, considerando evitar cambios hemodinámicos bruscos durante y después de la anestesia. Tener en cuenta el uso adecuado de uterotónicos, la administración debe ser individualizada según sea el caso y considerar medidas mecánicas o farmacológicas de tromboprofilaxis en este tipo de pacientes. La anestesia combinada fue segura y efectiva para esta paciente.

Agradecimientos: Agradecer a las personas que hicieron posible la realización y culminación del presente caso clínico y revisión de la literatura.

\section{Referencias}

1. Gómez RR, Nazar CJ. Consideraciones generales de la embarazada con enfermedad cardiaca congénita y adquirida. Rev Chil Anest. 2013;42(1):77-87.

2. Múnera-Echeverri AG. Heart disease and pregnancy. Rev Colomb Cardiol [Internet]. 1 de enero de 2018 [citado 9 de agosto de 2020];25:49-58. Disponible en: http://www.elsevier.es/es-revistarevista-colombiana-cardiologia-203-articulo-enfermedad-cardiaca-embarazo-S0120563317302589

3. Irina $Y$, Corredor P De, Elena L, Pérez C, Alberto J, Irina $Y$, et al. Cardiopatías congénitas y embarazo: Consideraciones anestésicas.
2001:2-16.

4. Schlichting LE, Insaf TZ, Zaidi AN, Lui GK, Van Zutphen AR. Maternal Comorbidities and Complications of Delivery in Pregnant Women With Congenital Heart Disease. J Am Coll Cardiol. 2019;73(17):2181-91.

5. Wijesingha S, White M. Anaesthetic implications of congenital heart disease for children undergoing non-cardiac surgery. Anaesth Intensive Care Med [Internet]. 2015;16(8):395-400. Disponible en: http://dx.doi.org/10.1016/j.mpaic.2015.06.002

6. Hollier L, Jr JNM, Connolly H, Turrentine M, Hameed A, Arendt KW, et al. Clinical management guidelines for obstetrician - gynecologists pregnancy and heart disease. Pract Bull Pregnancy Hear Dis. 2019;133(5):320-56

7. Socha NI, Echeverri A. Manejo anestésico de la paciente embarazada. Manual Práctico. Primera Ed. Rodas JC, editor. Medellin - Colombia: Universidad Pontificia Bolivariana; 2018. 185-186 p.

8. Pillutla P, Nguyen T, Markovic D, Canobbio M, Koos BJ, Aboulhosn JA. Cardiovascular and neonatal outcomes in pregnant women with high-risk congenital heart disease. Am J Cardiol. 15 de mayo de 2016;117(10):1672-7.

9. Regitz-Zagrosek V, Roos-Hesselink JW, Bauersachs J, BlomströmLundqvist C, Cífková R, De Bonis M, et al. 2018 ESC Guidelines for the management of cardiovascular diseases during pregnancy. European Heart Journal. 2018;3165-241.

10. Langesæter E, Dragsund M, Rosseland LA. Regional anaesthesia for a Caesarean section in women with cardiac disease: A prospective study. Acta Anaesthesiol Scand. 2010;54(1):46-54.

11. Heesen M, Carvalho B, Carvalho JCA, Duvekot JJ, Dyer RA, Lucas DN, et al. International consensus statement on the use of uterotonic agents during caesarean section. Anaesthesia. 2019;74(10):1305-19. 